\title{
NIVELES DE OOQUISTES DE EIMERIA EN CAMA Y SU RELACIÓN CON LAS LESIONES INTESTINALES EN POLLOS BROILER
}

\author{
María Salinas J. ${ }^{1}$, Eliana Icochea D. ${ }^{2}$, Eva Casas A. ${ }^{3}$, Néstor Falcón ${ }^{4}$ y Pablo Reyna ${ }^{1}$
}

\section{Eimeria oocyst levels and intestinal lesions in broiler chickens.}

Avian coccidiosis is a disease which causes important economic losses to avian production worldwide. The objective of this study were to determine the relationship between oocyst concentration in litter and grade of gut injury, as well as the incidence of subclinical coccidiosis. A total of 216 birds were studied between April and June 1999 , when temperatures and humidity fluctuated between $19-21^{\circ} \mathrm{C}$ and $60-85 \%$. Litter samples were collected using the double $W$ method at days $0,7,14,21,28,35,42$ of the birds life, and processed utilising the modified McMaster method. Macroscopic and microscopic evaluation of intestinal lesions was made when half the birds $(n=108)$ were sacrificed at 28 days of age, and the remainder $(n=108)$ at 35 days. Macroscopic injuries of grades +1 and +2 were observed in $7.7 \%$ of the 28 day old birds and $4.3 \%$ of the 35 day old birds, while microscopic injuries were found in $48.8 \%$ and $42 \%$ of the 28 and 35 day old animals. Although the frequency of oocysts per gram of litter was greatest between days 28 and 42 , the correlation with incidence of macro and microscopic lesions at days $28(0.748)$ and $35(0.432)$ was only moderate.

Key words: Coccidiosis, oocysts, intestinal lesions

La coccidiosis aviar es una enfermedad presente en todo el mundo que ocasiona grandes pérdidas económicas en la avicultura mundial. El presente estudio tuvo por objetivo determinar la relación entre concentración de ooquistes en cama y el grado de lesiones intestinales, así como aportar información sobre la situación actual de la coccidiosis subclínica en el Perú. El estudio fue realizado durante los meses de abril a junio de 1999 , cuando la temperatura ambiental estuvo entre 19 a $21^{\circ} \mathrm{C}$ y la humedad relativa entre $60-85 \%$. Se obtuvieron muestras de cama por el método de la doble $W$ a los $0,7,14,21,28,35,42,49$ días de edad y fueron procesadas por el método de Mc Master modificado. Se sacrificaron 108 aves a los 28 y 35 días de edad respectivamente, realizándose la evaluación de lesiones intestinales macroscópicas y microscópicas. En el recuento de ooquistes por gramo de cama el mayor grado de contaminación de ooquistes se presentó entre 4 y 6 semanas de edad. De las aves sacrificadas a los 28 y 35 días se encontró que el $7.7 \%$ y $4.3 \%$ de las mismas presentaron lesiones macroscópicas cuyos grados fueron +1 y +2 ; mientras que el $48.8 \%$ y $42 \%$, presentaron lesiones microscópicas. El $51.2 \%$ y $58 \%$, fueron negativas. La correlación entre el grado de contaminación de ooquistes en cama y el grado de lesiones macro y microscópicas entre los 28 y 35 días de edad fue de grado moderado (0.748 y 0.432$)$.

Palabras clave: Coccidiosis, ooquistes, lesiones.

1 Práctica Privada

${ }^{2}$ Laboratorio de Patología Aviar y Producción Avícola UNMSM-FMV

${ }^{3}$ Laboratorio de Parasitología Veterinaria UNMSM-FMV

4 Laboratorio de Medicina Veterinaria Preventiva UNMSM-FMV 
La coccidiosis aviar es una enfermedad parasitaria cosmopolita causada por protozoos del Phylum Apicomplexa, familia Eimeriidae, género Eimeria que se produce mediante la ingestión de ooquistes esporulados causando procesos clínicos o subclínicos.

Después de mųchos años de conocer los diferentes aspectos de la coccidiosis aviar, ésta continúa siendo en la avicultura, y muy especialmente en la producción de pollos broiler, la causante de enormes pérdidas económicas, atribuyéndose a una pérdida mundial anual de 250 millones de dólares.

Las lesiones macroscópicas producidas por las diferentes especies de coccidia son muy típicas y están relacionadas con la severidad del brote.

La administración continua de anticoccidiales en el alimento redujo en las últimas dos décadas la intensidad de las presentaciones clínicas de la enfermedad, dando lugar a la aparición de casos más leves que reciben el nombre de coccidiosis subclínica, los que representan un riesgo permanente para el buen rendimiento productivo de los animales. Esta última condición ha recibido también el nombre de "Coccidiasis" la que indica una infección que no produce una alteración en los parámetros productivos fácilmente detectable.

El recuento de ooquistes por gramo de cama es usado para evaluar de una manera indirecta el comportamiento de los anticoccidiales en los planteles de crianza, como parte de un programa de evaluación anticoccidial utilizándose por otro lado la observación de lesiones macroscópicas y microscópicas del tracto intestinal, sin embargo, en nuestro país esta prueba sólo se hace cuando se detectan en las aves problemas en parámetros productivos tales como pigmentación, ganancia de peso y conversión alimenticia.
El objetivo del presente estudio fue el de determinar la relación entre la concentración de ooquistes en cama y los grados de las lesiones intestinales, así como aportar información sobre la situación actual de la coccidiosis subclínica en el país.

El presente trabajo se realizó en seis planteles de pollos de carne de la Integración Avícola San Fernando ubicadas en la zona de Ventanilla, Huaral y Huacho. Cada plantel tenía 10 galpones y en cada galpón había un promedio de 15,000 aves.

La fase experimental se realizó durante los meses de abril, mayo y junio de 1999 ; la temperatura ambiental registrada osciló entre los $19-21^{\circ} \mathrm{C}$ y la humedad relativa entre $60-85 \%$.

En el presente estudio se usaron seis granjas y 18 galpones, trabajando con los planteles centinelas de la Integración Avícola San Fernando.

Se tomaron muestras de cama de seis planteles, seleccionándose al azar tres galpones en cada uno de ellos, lo que hizo un total de 18 galpones, con un promedio de 15,000 aves por galpón.

Las muestras fueron tomadas desde el día 0 y los días 7, 14, 21, 28, 35, 42, 49 de la campaña, siguiendo el método de la doble $\mathrm{W}$. (Rojas, 1990).

Se tomaron al azar 6 pollos por galpón, a los 28 y 35 días de edad, de los mismos galpones que fueron seleccionados para la toma de muestras de cama, ya que entre los 28 y 35 días se encuentran los mayores niveles de contaminación de la cama, haciendo un total de 216 aves, para el estudio macro y microscópico de las lesiones intestinales. 
Para el procesamiento de las muestras de camas se empleó el método de Mc Master modificado para cama de galpón de aves (Rojas, 1990).

El número de ooquistes contabilizados en un recuadro de la cámara se multiplicó por el factor $100 \mathrm{y}$ los resultados fueron expresados en número de ooquistes/g de cama.

Las aves fueron sacrificadas a los 28 y 35 días de edad para observar las lesiones macro y microscópicas del tracto intestinal.

El examen macroscópico consistió en la observación de la serosa, mucosa y contenido intestinal, buscando las alteraciones anatomopatológicas a nivel intestinal siguiendo la técnica desarrollada por Johnson y Reid (1970), quienes idearon una escala según el grado de lesiones que va de +0 hasta +4 .

$$
\begin{aligned}
& +0=\text { Normal } \\
& +1=\text { Infección ligera } \\
& +2=\text { Infección moderada } \\
& +3=\text { Infección grave } \\
& +4=\text { Infección muy grave, con mortali- } \\
& \text { dad }
\end{aligned}
$$

El examen microscópico se realizó mediante el raspado de la mucosa del duodeno, tercio medio del intestino y ciegos (Johnson y Reid, 1970).

La observación de los ooquistes se hizo en el microscopio óptico entre porta y cubre objeto, recorriendo los preparados en su totalidad (Franceschi y Mattiello, 1995).

Los análisis del grado de contaminación y el score de lesiones macroscópicas y microscópicas fueron hechos por la prueba de correlación de Spearman y por el Test Mann-Whitney.

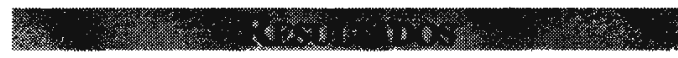

En el Cuadro 1 se observa un comportamiento similar en 4 de los planteles evaluados. El día 28 mostró la máxima concentración de ooquistes empezando a disminuir posteriormente. Los dos planteles restantes presentaron el pico de incremento de concentración de ooquistes el día 42 para decaer posteriormente.

Cuadro 1. Recuento de Ooquistes de Eimeria en cama de los planteles de pollos Broiler de la zona de Ventanilla, Huaral, Huacho durante los meses de abril a junio de 1999.

\begin{tabular}{ccccccccc}
\hline PlanteVíá & \multicolumn{7}{c}{ n de ooquistes/g cama } \\
\cline { 2 - 8 } & 0 & 7 & 14 & 21 & 28 & 35 & 42 & 49 \\
\hline 1 & 50 & 33 & 200 & 583 & 1883 & 1733 & 1152 & 600 \\
2 & 717 & 417 & 583 & 1117 & 2067 & 2167 & 3516 & 2450 \\
3 & 50 & 100 & 567 & 1533 & 3200 & 2217 & 1217 & 750 \\
4 & 117 & 133 & 433 & 1133 & 2217 & 2083 & 1200 & 717 \\
5 & 0 & 83 & 317 & 1016 & 1450 & 3917 & 4450 & 1500 \\
6 & 67 & 217 & 550 & 1417 & 3400 & 2250 & 1683 & 783 \\
\hline
\end{tabular}


En el cuadro 2 se muestra los resultados del score de lesiones intestinales macroscópicas y microscópicas de las aves. Se encontró que $92.3 \%$ de las aves fueron negativas al examen macroscópico, mientras que $51.2 \%$ de las mismas muestras negativas fueron negativas al examen microscópico. El $7.7 \%$ de las muestras presentaron lesiones macroscópicas, $6.8 \%$ de grado $1 \mathrm{y}$ $0.9 \%$ de grado 2 .

Si bien sólo el $7.7 \%$ de las muestras presentó lesiones macroscópicas el $48.8 \%$ de las mismas presentó lesiones microscópicas, siendo el $39.5 \%$ de grado 1 y el $9.3 \%$ de grado 2 .

El Cuadro 3 muestra los resultados del score de lesiones macroscópicos y microscópicos de las aves sacrificadas a los 35 días de edad. Se encontró que el $95.7 \%$ fueron negativas al examen macroscópico. El 4.3\% presentaron lesiones macroscópicas, $3.7 \%$ de grado 1 y $0.6 \%$ de grado 2 .

Microscópicamente se encontró que un $42 \%$ de las muestras fueron positivas a presencia de ooquistes; de éstas $34.6 \%$ de grado 1 y el $7.4 \%$ de grado 2 .

Así también, se encontró lesiones microscópicas de $E$. acervulina de grado +1 y +2 ; de $E$. tenella de grado +1 y +2 . En el raspado de mucosa del tercio medio del intestino se halló ooquistes de E. maxima los cuales se diferencian por su gran tamaño.

Mediante la prueba de Mann-Whitney se determinó que no existen diferencias entre las lesiones macroscópicas de duodeno, tercio medio del intestino y el ciego a los 28 y 35 días de edad; así mismo no se halló diferencias en el examen microscópico del raspado de la mucosa del ciego y del tercio medio del intestino. Donde si se encontró diferencia ( $p<0.001$ ) fue al examen microscópico del raspado de mucosa del duodeno.

La correlación entre el grado de contaminación de ooquistes en la cama y las lesiones intestinales macro y microscópicas entre los días 28 y 35 fueron de 0.748 y 0.432 respectivamente. Estas correlaciones fueron consideradas como moderadas.

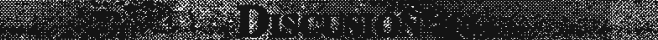

En el presente estudio se tomaron muestras de cama un día antes de introducir el nuevo lote, encontrándose ooquistes de Eimeria $s p$ en todos los galpones evaluados en diferentes concentraciones lo cual es consecuencia de la crianza en piso en de tierra y a las óptimas condiciones ambientales para la supervivencia de ooquistes (Shirley, 1994).

El pico de la concentración de eimeria se presentó entre las 4 a 6 semanas disminuyendo posteriormente; similar a lo encontrado por Long et al. (1975), quienes hallaron que el número de ooquistes es bajo y variable a las dos semanas de haber introducido los pollos, probablemente debido a la presencia, en esa etapa, de pequeña cantidad de materia fecal, la que alcanza su pico a las 4-6 semanas y disminuye a las 8-9 semanas. Igualmente Reyna (Comunicación personal) afirma que durante las primeras semanas la concentración de ooquistes en la cama se mantiene baja porque no cuenta con las condiciones de humedad necesaria para la esporulación y de otro lado por la inmadurez del sistema digestivo de las aves quienes ingieren ooquistes pero no cuentan con las enzimas para la liberación de los esporozoitos necesaria para su reproduccción.

La relación encontrada entre el grado de contaminación de la cama y las lesiones intestinales fue moderada y esto puede ser debido a que son muchos los factores que interactúan en la presentación de coccidiosis clínica y también a la variedad de especies de coccidias presentes con sus diferentes potenciales reproductivos, tal como fue expresado por Mc Dougald (2000). Así mismo esta relación también puede verse afectada por el uso constante de los anticoccidiales, que influyen directamente en el desarrollo de las lesiones por coccidias. 
Cuadro 2. Número de animales afectados por los diferentes grados de lesiones intestinales coccidiales a los 28 días de edad, de los planteles de Ventanilla, Huaral, Huacho durante los meses de abril a junio de 1999.

\begin{tabular}{|c|c|c|c|c|c|c|c|}
\hline \multirow{2}{*}{ Plantel } & \multirow{2}{*}{ Intestino } & \multicolumn{2}{|c|}{ Negativos } & \multicolumn{2}{|c|}{ Grado 1} & \multicolumn{2}{|r|}{ Grado 2} \\
\hline & & Macro & Micro & Macro & Micro & Macro & Micro \\
\hline & Duodeno & 16 & 9 & 2 & 7 & 0 & 2 \\
\hline \multirow[t]{3}{*}{1} & Intestino medio & 18 & 11 & 0 & 7 & $\mathbf{0}$ & 0 \\
\hline & Ciego & 17 & 10 & 1 & 6 & 0 & 2 \\
\hline & Duodeno & 18 & 7 & 0 & 11 & 0 & 0 \\
\hline \multirow[t]{3}{*}{2} & Intestino medio & 18 & 15 & 0 & 3 & 0 & 0 \\
\hline & Ciego & 17 & 12 & 1 & 5 & $\mathbf{0}$ & 1 \\
\hline & Duodeno & 13 & 11 & 4 & 3 & 1 & 4 \\
\hline \multirow[t]{3}{*}{3} & Intestino medio & 18 & 7 & 0 & 11 & 0 & 0 \\
\hline & Ciego & 15 & 9 & 3 & 7 & 0 & 2 \\
\hline & Duodeno & 18 & 7 & 0 & 11 & 0 & 0 \\
\hline \multirow[t]{3}{*}{4} & Intestino medio & 18 & 7 & $\mathbf{0}$ & 9 & 0 & 2 \\
\hline & Ciego & 17 & 8 & 1 & 9 & 0 & 1 \\
\hline & Duodeno & 18 & 7 & $\mathbf{0}$ & 11 & 0 & 0 \\
\hline \multirow[t]{3}{*}{5} & Intestino medio & 18 & 9 & 0 & 9 & 0 & 0 \\
\hline & Ciego & 18 & 12 & 0 & 6 & 0 & 0 \\
\hline & Duodeno & 11 & 3 & 7 & 7 & 0 & 8 \\
\hline \multirow[t]{2}{*}{6} & Intestino medio & 18 & 13 & 0 & 3 & 0 & 2 \\
\hline & Ciego & 13 & 9 & 3 & 3 & 2 & 6 \\
\hline & Total & 299 & 166 & 22 & 128 & 3 & 30 \\
\hline & $\%$ & 92.3 & 51.2 & 6.8 & 39.5 & 0.9 & 9.3 \\
\hline
\end{tabular}

Cuadro 3. Número de animales afectados por los diferentes grados de lesiones intestinales coccidiales a los 35 días de edad, de los planteles de Ventanilla, Huaral, Huacho durante los meses de abril a junio de 1999.

\begin{tabular}{|c|c|c|c|c|c|c|c|}
\hline \multirow{2}{*}{ Plantel } & \multirow{2}{*}{ Intestino } & \multicolumn{2}{|c|}{ Negativos } & \multicolumn{2}{|c|}{ Grado 1} & \multicolumn{2}{|c|}{ Grado 2} \\
\hline & & Macro & Micro & Macro & Micro & Macro & Micro \\
\hline \multirow{4}{*}{1} & Duodeno & 18 & 18 & 0 & 0 & 0 & 0 \\
\hline & Intestino medio & 18 & 15 & 0 & 3 & 0 & 0 \\
\hline & Ciego & 18 & 16 & 0 & 2 & 0 & 0 \\
\hline & Duodeno & 18 & 10 & 0 & 8 & 0 & 0 \\
\hline \multirow[t]{3}{*}{2} & Intestino medio & 18 & 13 & 0 & 5 & 0 & 0 \\
\hline & Ciego & 16 & 2 & 1 & 14 & 1 & 2 \\
\hline & Duodeno & 18 & 15 & 0 & 3 & 0 & 0 \\
\hline \multirow[t]{3}{*}{3} & Intestino medio & 18 & 12 & $\mathbf{0}$ & 6 & 0 & 0 \\
\hline & Ciego & 16 & 9 & 2 & 7 & 0 & 2 \\
\hline & Duodeno & 18 & 13 & 0 & 5 & 0 & 0 \\
\hline \multirow[t]{3}{*}{4} & Intestino medio & 18 & 11 & 0 & 5 & 0 & 2 \\
\hline & Ciego & 17 & 9 & 1 & 6 & 0 & 3 \\
\hline & Duodeno & 10 & 1 & 7 & 9 & 1 & 8 \\
\hline \multirow[t]{3}{*}{5} & Intestino medio & 18 & 12 & 0 & 6 & 0 & 0 \\
\hline & Ciego & 17 & 7 & 1 & 10 & 0 & 1 \\
\hline & Duodeno & 18 & 12 & 0 & 5 & 0 & 1 \\
\hline \multirow[t]{4}{*}{6} & Intestino medio & 18 & 10 & 0 & 7 & 0 & 1 \\
\hline & Ciego & 18 & 3 & 0 & 11 & 0 & 4 \\
\hline & Total & 310 & 188 & 12 & 112 & 2 & 24 \\
\hline & $\%$ & 95.7 & 58 & 3.7 & 34.6 & 0.6 & 7.4 \\
\hline
\end{tabular}


La diferencia significativa encontrada en el examen del raspado de mucosa de duodeno, puede deberse al grado de lesión que produce la $E$. acervulina la cual es leve y posiblemente permite una rápida regeneración de los tejidos afectados.

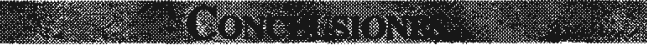

- Todos los planteles presentaron diferentes grados de contaminación de ooquistes en la cama. El nivel máximo de concentración se obtuvo entre los 28 y 42 días de edad de las aves.

- Las aves de todos los planteles estudiados presentaron lesiones microscópicas en grados variables, mientras que sólo un $7.7 \%$ y $4.3 \%$ presentaron lesiones macroscópicas a los 28 y 35 días de edad, respectivamente, lo que refleja la presencia constante de coccidiosis subclínica en los planteles de crianza de pollos broiler.

- Se encontró relación moderada entre el grado de contaminación de la cama y las lesiones intestinales.

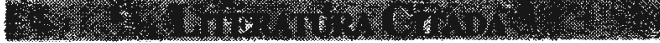

1. Franceschi. M., R. Mattiello. 1995. Diagnóstico de la coccidiosis subclínica por el método de raspajes seriados, XIV Congreso Latinoamericano de Avicultura. Chile. p. 10-13.

2. Johnson. J., W. N. Reid. 1970. Anticoccidial drugs: lesion scoring technics in battery and floor-pen experiments with chickens, Experimental Parasitology. 28: 30-36.

3. Long. P. L., R. Tompkins \& B. J. Millard. 1975. Coccidiosis in broilers; evaluation of infection by the examination of broiler house litter for oocystos. Avian Pathol 4: 297-289.

4. Mc Dougald. L. 2000. E-mail: lrmed @arches.uga.edu

5. Rojas. M. 1990. Parasitismo de los rumiantes domésticos; terapia, prevención y modelos para su aprendizaje. 1a. ed., p.368-377. Ed. Maijosa, Perú

6. Shirley. M. 1994. Epizootiología. Simposio internacional coccidiose. Brazil, p. 11-22. 